\title{
Perfil de Segurança dos Stents Farmacológicos nas Síndromes Coronárias Agudas: Dados do Registro INCOR
}

\author{
Carlos Augusto Homem de Magalhães Campos ${ }^{1}$, Expedito E. Ribeiro', Pedro A. Lemos ${ }^{1}$, João L. Falcão, \\ Andre G. Spadaro ${ }^{1}$, Luiz J. Kajita ${ }^{1}$, Antonio Esteves Filho ${ }^{1}$, Marco A. Perin ${ }^{1}$, Pedro E. Horta ${ }^{1}$, \\ Marcus N. Gamaํ, Gilberto Marchiori¹, Eulógio Martinez ${ }^{1}$
}

\section{RESUMO}

Fundamentos: Os stents farmacológicos constituem um grande avanço no tratamento da doença coronária. No entanto, seu emprego nas síndromes coronárias agudas tem sido objeto de intensa discussão científica. Método: Entre maio de 2002 e setembro de 2006, 910 pacientes consecutivos foram tratados com implante de pelo menos um stent farmacológico e incluídos na presente análise. Os pacientes foram divididos em dois grupos: grupo estável (635 portadores de angina estável) e grupo agudo (275 pacientes com síndrome coronária aguda sem supra de ST). Analisamos as características clínicas e angiográficas e a ocorrência tardia de eventos adversos. Resultados: As características clínicas foram semelhantes, exceto pela maior freqüência de tabagistas nos instáveis e de intervenção percutânea prévia nos estáveis. Após 588 dias (mediana do seguimento), os grupos, estável e agudo, tiveram índices semelhantes de reinfarto $(2,8 \%$ vs. 5,0\%; $p=0,1)$, revascularização do vaso-alvo $(6,0 \%$ vs. $7,7 \%$; $p=0,4)$, óbito $(4,5 \%$ vs. $6,5 \% ; p=0,2)$ e eventos cardíacos maiores combinados $(9,9 \%$ vs. $11,9 \%$; $p=0,4)$, respectivamente. No entanto, a ocorrência de trombose foi significativamente mais freqüente nos pacientes com quadros coronários agudos $(1,4 \%$ vs. $4,4 \% ; p=0,02)$, principalmente pela ocorrência de trombose ao longo do primeiro ano após implante $(1,1 \%$ vs. $4,4 \% ; p=0,01)$. Conclusão: Os stents farmacológicos demonstraram bom perfil de segurança nos pacientes com síndromes coronárias agudas em relação àqueles portadores de quadros clínicos estáveis, apesar da maior ocorrência de trombose tardia do stent.

DESCRITORES: Stents farmacológicos. Síndrome coronariana aguda. Trombose.

\section{SUMMARY}

Drug Eluting Stents Safety in Acute Coronary Syndromes: The INCOR Registry

Background: Drug-eluting stents are a great advance in the treatment of coronary disease. However, their use in patients with acute coronary syndromes has been the subject of intense scientific debate. Methods: 910 consecutive patients treated with at least one drug-eluting stent between May 2002 and September 2006 were enrolled in the present analysis. The patients were assigned to 2 groups according to their clinical condition at the time of admission: 1) Stable group (635 patients with stable angina) and 2) Acute group (275 patients with NSTEMI). We analyzed the clinical and angiographic characteristics as well as the occurrence of late adverse events. Results: The clinical characteristics of the groups were similar, except for the highest incidence of smokers in the acute group and previous percutaneous intervention in the stable group. After 588 days (median follow-up period), the stable and acute groups had similar rates of re-infarction (2.8 vs. $5.0 \% ; \mathrm{p}=0.1)$, target vessel revascularization (6.0 vs. $7.7 \% ; p=0.4)$, death ( 4.5 vs. $6.5 \% ; p=0.2)$ and composite major adverse cardiac events (9.9 vs. $11.9 \%$; $p=0.4$ ), respectively. However, the occurrence of in-stent thrombosis was more frequent in patients with acute coronary diseases ( 1.4 vs. $4.4 \% ; p=0.02$ ), mainly due to the occurrence of thrombosis within the first year after implantation (1.1 vs. $4.4 \% ; p=0.01)$. Conclusion: Drug-eluting stents have shown a good safety profile in patients with acute coronary syndromes compared to those with chronic coronary disease, despite the higher incidence of late in-stent thrombosis.

DESCRIPTORS: Drug-eluting stents. Acute coronary syndrome. Thrombosis. Major adverse cardiac event.

O stents farmacológicos já demonstraram superioridade quanto à redução de reestenose coronária, quando comparados aos stents metálicos convencionais, em diversos estudos randomizados com milhares de pacientes ${ }^{1-9}$. Em decorrência dessa eficácia, passaram a ser utilizados no tratamento da doença arterial coronária em diversos subgrupos,

Medicina da Universidade de São Paulo - São Paulo, SP.

Correspondência: Carlos Augusto Homem de Magalhães Campos.

Av. Heitor Penteado, 250 - apto. 73 - Sumarezinho - São Paulo, SP

- CEP 05438-000

E-mail: cm-campos@uol.com.br

Recebido em: 2/7/2008 • Aceito em: 3/9/2008 
em cenários distintos daqueles encontrados nos protocolos clínicos. Entre esses subgrupos, a presença de síndromes coronárias agudas ainda é motivo de questionamentos na indicação de stents eluidores por estar associada à ocorrência de trombose do stent ${ }^{10}$. Nesse contexto, buscamos avaliar o impacto do quadro clínico à admissão sobre a ocorrência de eventos adversos em uma série de pacientes consecutivos tratados com stents farmacológicos em um centro brasileiro de atenção médica terciária.

\section{MÉTODO}

Incluímos neste estudo, prospectiva e consecutivamente, todos os pacientes tratados entre maio de 2002 e setembro de 2006 com pelo menos um stent coronário farmacológico, com sucesso angiográfico (ou seja, estenose residual ao término do procedimento $<30 \%$ à análise visual), no Serviço de Hemodinâmica e Cardiologia Intervencionista do Instituto do Coração (InCor) do Hospital das Clínicas da Faculdade de Medicina da Universidade de São Paulo, na cidade de São Paulo. Não houve qualquer restrição para inclusão quanto a quadro clínico à admissão, idade, disfunção ventricular, características angiográficas ou presença de co-morbidades.

Os pacientes foram divididos em dois grupos, de acordo com o quadro clínico à admissão:

- Grupo agudo: pacientes com angina do peito com características instáveis (dor em repouso ou progressiva), desde que não houvesse supradesnível persistente do segmento ST superior a $1 \mathrm{mV}$ em duas derivações contíguas ou bloqueio de ramo esquerdo novo. Foram coletados marcadores de necrose miocárdica (CKMB massa e troponina l) para diagnóstico de infarto agudo do miocárdio sem supradesnível de ST.

- Grupo estável: portadores de angina estável, equivalente anginoso ou assintomáticos com isquemia silenciosa crônica demonstrada por prova funcional.

O objetivo primário foi comparar a ocorrência de eventos adversos cardíacos maiores combinados (mortalidade global, infarto e revascularização do vasoalvo) ao longo do tempo entre os grupos. Foi também analisada a ocorrência de trombose do stent, classificada de acordo com os critérios do Academic Research Consortium $^{11}$.

Assim, a classificação quanto ao tempo de ocorrência foi a seguinte:

a) aguda - ocorrida dentro das primeiras 24 horas;

b) subaguda - entre o primeiro e o trigésimo dias após implante;

c) tardia - do trigésimo dia até o final do primeiro ano após implante; e

d) muito tardia - após o primeiro ano do implante.
E quanto ao grau de certeza:

a) definitiva - confirmação angiográfica ou por meio de necropsia de trombose no vaso-alvo;

b) provável - qualquer óbito não explicado nos primeiros trinta dias após o procedimento ou qualquer infarto do miocárdio no território relacionado ao vaso tratado, sem confirmação angiográfica; e

c) possível - qualquer óbito não explicado após trinta dias do implante.

Os dados da evolução foram coletados por médicos treinados, seguindo o preenchimento de formulários previamente padronizados. A coleta incluiu características clínicas, resultados de exames laboratoriais, dados do procedimento invasivo (desde tempo até características angiográficas e da intervenção) e evolução clínica tardia. A coleta de informações sobre a evolução tardia foi realizada por seguimento ambulatorial realizado em nossa instituição, revisão dos registros hospitalares ou contato telefônico.

A angiografia coronária quantitativa foi realizada utilizando um sistema computadorizado de detecção de bordas (CASS II, Pie Medical, Maastricht, Holanda). Também classificamos as lesões tratadas como sendo complexas se houvesse a presença de um dos critérios já bem estabelecidos previamente ${ }^{12}$ : defeito intraluminal consistente com trombo, definido como fechamento abrupto do vaso com retenção de contraste ou defeito de enchimento intraluminal de um vaso patente adjacente a uma lesão estenótica em torno de uma região com opacificação homogênea de contraste; ulceração da placa, definida pela presença de contraste e contornos imprecisos além do lúmen vascular; irregularidade da placa, definida por bordas irregulares ou sobrepostas; e diminuição de fluxo.

\section{Análise Estatística}

As variáveis contínuas foram descritas como média \pm desvio padrão e comparadas entre os grupos com o teste $t$ de Student. As variáveis categóricas foram representadas como sua porcentagem e comparadas entre os grupos com o teste exato de Fischer. As características demográficas, clínicas e angiográficas foram testadas em um modelo de análise multivariada de Cox para identificar preditores de mortalidade entre os grupos. Todas as características listadas na Tabela 1 foram testadas neste modelo de regressão logística.

\section{RESULTADOS}

Nos primeiros quatro anos de nossa série de stents eluidores, tratamos 910 pacientes com 1.230 stents farmacológicos. Nesse grupo, os stents farmacológicos utilizados, em ordem de freqüência, foram: Cypher ${ }^{\circledR}$ (41\%), Taxus ${ }^{\circledR}(36 \%)$, Infinnium $^{\circledR}(22 \%)$, CoStar $^{\circledR}(3 \%)$, Supralimus ${ }^{\circledR}(2 \%)$ e Endeavor ${ }^{\circledR}(0,5 \%)$. Vale ressaltar que $14 \%$ dos pacientes receberam também pelo me- 
nos um stent convencional. Desse total, 625 pacientes foram admitidos com síndromes coronárias estáveis e outros 275, com angina instável/infarto do miocárdio sem supradesnível de ST. No grupo agudo, o diagnóstico de infarto à admissão esteve presente em $37,8 \%$ dos pacientes. Obtivemos seguimento de 97,5\% dos pacientes por uma mediana de 588 dias (intervalo interquartil: 291-806 dias).

A média de idade foi de 64 anos, sendo mais de $70 \%$ dos pacientes do sexo masculino (Tabela 1). Aproximadamente $10 \%$ apresentavam disfunção ventricular, mais de $30 \%$ eram diabéticos e a maior parte dos pacientes apresentava doença coronária multiarterial (Tabela 1). As únicas diferenças clínicas entre os gru- pos foram a presença de tabagistas atuais $(7,3 \%$ vs. $18,7 \% ; p<0,01)$ no grupo agudo e a maior freqüência de pacientes com intervenção coronária percutânea prévia $(32,4 \%$ vs. $25,1 \% ; p=0,03)$ no grupo estável (Tabela 1). A duração da terapia antiplaquetária dupla, também semelhante entre as amostras, foi de cerca de 180 dias.

As características angiográficas, no entanto, revelaram diferenças significativas entre os grupos (Tabela 1). Naqueles pacientes com síndromes coronárias agudas, os vasos tinham menor diâmetro luminal mínimo $(0,9 \pm 0,3 \mathrm{~mm}$ vs. $0,8 \pm 0,3 \mathrm{~mm} ; \mathrm{p}<0,01)$ com maior porcentual de obstrução luminal $(64,8 \pm 10 \%$ vs. $68,7 \pm 10,5 \% ; p<0,01)$, apesar de possuírem maior

TABELA 1

Características clínicas e angiográficas da população do presente estudo

\begin{tabular}{|c|c|c|c|}
\hline & $\begin{array}{l}\text { Grupo estável } \\
\quad n=635\end{array}$ & $\begin{array}{c}\text { Grupo agudo } \\
n=275\end{array}$ & $\mathbf{p}$ \\
\hline Idade, anos & $63,9 \pm 11$ & $63,9 \pm 12$ & 0,19 \\
\hline Sexo masculino (\%) & $465(73,2)$ & $211(76,7)$ & 0,3 \\
\hline Hipertensão arterial (\%) & $493(78,8)$ & $215(78,8)$ & 1 \\
\hline Dislipidemia (\%) & $478(79,4)$ & $202(78,3)$ & 0,7 \\
\hline Diabetes melito (\%) & $219(34,9)$ & $82(30,4)$ & 0,2 \\
\hline Tabagismo atual (\%) & $45(7,3)$ & $50(18,7)$ & $<0,01$ \\
\hline $\operatorname{AVC}(\%)$ & $29(4,6)$ & $9(3,3)$ & 0,5 \\
\hline IRC dialítica (\%) & $3(0,5)$ & $2(0,7)$ & 0,6 \\
\hline IVP $(\%)$ & $21(3,4)$ & $6(2,2)$ & 0,4 \\
\hline IAM prévio (\%) & $163(27,4)$ & $86(33,1)$ & 0,1 \\
\hline $\operatorname{ICC}(\%)$ & $69(11,3)$ & $26(9,6)$ & 0,5 \\
\hline ICP prévia (\%) & $200(32,4)$ & $68(25,1)$ & 0,03 \\
\hline RM prévia (\%) & $118(19,1)$ & $62(22,9)$ & 0,2 \\
\hline Tempo de terapia antiplaquetária dupla, dias & $180(150-330)$ & $180(155-330)$ & 0,9 \\
\hline Padrão de DAC (\%) & & & 0,2 \\
\hline 1 vaso & $245(39,7)$ & $86(33,3)$ & \\
\hline 2 vasos & $196(31,8)$ & $88(34,1)$ & \\
\hline 3 vasos & $176(28,5)$ & $84(32,6)$ & \\
\hline \multicolumn{4}{|l|}{ Vaso tratado $(\%)$} \\
\hline CD & $215(33,9)$ & $91(33,1)$ & 0,9 \\
\hline TCE & $19(3)$ & $5(1,8)$ & 0,4 \\
\hline DA & $345(54,3)$ & $152(55,3)$ & 0,8 \\
\hline $\mathrm{CX}$ & $181(28,5)$ & $72(26,2)$ & 0,5 \\
\hline Número de lesões tratadas & $1,6 \pm 0,8$ & $1,7 \pm 0,9$ & 0,8 \\
\hline Diâmetro de referência, mm & $2,5 \pm 0,44$ & $2,6 \pm 0,52$ & $<0,01$ \\
\hline $\mathrm{DLM}, \mathrm{mm}$ & $0,9 \pm 0,31$ & $0,8 \pm 0,32$ & $<0,01$ \\
\hline Estenose, \% & $64,8 \pm 10$ & $68,7 \pm 10,5$ & $<0,01$ \\
\hline Número de stents utilizados & $1,5 \pm 0,8$ & $1,6 \pm 0,9$ & 0,1 \\
\hline Lesões complexas (Ambrose), \% & 18,3 & 62,1 & $<0,01$ \\
\hline
\end{tabular}


diâmetro de referência $(2,5 \pm 0,4 \mathrm{~mm}$ vs. $2,6 \pm 0,5 \mathrm{~mm}$; $p<0,01)$. O grupo agudo também demonstrou maior ocorrência de lesões complexas em relação ao grupo estável (18,3\% vs. 62,1\%; p < 0,01).

Conforme ilustrado na Tabela 2, após seguimento médio de 588 dias, a ocorrência de eventos cardíacos adversos maiores - uma combinação de óbito, infarto e revascularização do vaso-alvo - foi de 9,9\% no grupo estável e de 11,9\% no grupo agudo, não atingindo significância estatística $(p=0,4)$. De maneira semeIhante, não houve diferença significante, entre os grupos estável e agudo, com relação à ocorrência de óbito $(4,5 \%$ vs. $6,5 \%$; $p=0,2)$, infarto $(2,8 \%$ vs. $5,0 \%$; $\mathrm{p}=0,1)$ e revascularização do vaso-alvo $(6,0 \%$ vs. $7,7 \%$; $p=0,4)$, respectivamente.

No entanto, a incidência global de trombose de stent foi significativamente maior no grupo de pacientes tratados com síndromes coronárias agudas sem supradesnível do segmento ST (1,4\% vs. 4,4\%; $p=0,02)$. Essa diferença pôde ser notada principalmente pela maior ocorrência de trombose no primeiro ano após o implante $(1,1 \%$ vs. $4,0 \% ; p=0,01)$ no grupo agudo (Tabela 2).

Foram identificados como preditores independentes de mortalidade, à análise multivariada: idade, presença de diabetes melito, doença arterial coronária triarterial, insuficiência cardíaca congestiva e presença de insuficiência renal requerendo diálise (Tabela 3).

\section{DISCUSSÃO}

Os principais achados deste estudo sobre a terapêutica da doença arterial coronária com stents farmacológicos, em pacientes do "mundo real", na presença de angina instável/infarto agudo do miocárdio sem supradesnível de ST foram que: (1) o grupo estável teve características clínicas semelhantes, mas com lesões menos complexas à angiografia; (2) a presença do quadro agudo não incrementou a ocorrência de eventos cardíacos adversos maiores em um segmento tardio; (3) os pacientes das síndromes instáveis estiveram expostos a maior índice de trombose dos stents eluidores ao longo do primeiro ano.

Apesar de se tratar de um estudo não-randomizado, os grupos analisados apresentaram características relativamente homogêneas. Entre os pacientes agudos, a maioria $(62,2 \%)$ era de portadores de angina instável e, portanto, não apresentava elevação de marcadores de necrose miocárdica. No entanto, à análise angiográfica, esse grupo fica bem caracterizado como agudo, já que 62,1\% possuíam características de lesões complexas. Nesse ponto, houve acentuada diferença, já que os pacientes com síndromes estáveis só apresentaram esse tipo de lesão em 18,3\%. Na literatura, está bem documentado que a presença de lesões complexas tem relação direta com a gravidade da angina pela classificação de Braunwald ${ }^{13,14}$.
TABELA 2

Ocorrência de eventos de acordo com o grupo estudado

\begin{tabular}{lccc}
\hline & $\begin{array}{c}\text { Grupo estável } \\
\mathbf{n}=\mathbf{6 3 5}\end{array}$ & $\begin{array}{c}\text { Grupo agudo } \\
\mathbf{n}=\mathbf{2 7 5}\end{array}$ \\
\hline ECAM $(\%)$ & 9,9 & 11,9 & 0,4 \\
IAM $(\%)$ & 2,8 & 5 & 0,1 \\
RVA $(\%)$ & 6 & 7,7 & 0,4 \\
Óbito $(\%)$ & 4,5 & 6,5 & 0,2 \\
Qualquer trombose $(\%)$ & 1,4 & 4,4 & 0,01 \\
Definitiva & 0,6 & 2,2 & 0,04 \\
Possível & 0,4 & 1,1 & 0,2 \\
Provável & 0,4 & 1,1 & 0,2 \\
Trombose por tempo (\%) & & & 0,02 \\
Aguda & 0 & 0 & 1 \\
Subaguda & 0,5 & 1,1 & 0,4 \\
Tardia & 0,6 & 2,9 & 0,01 \\
Muito tardia & 0,3 & 0,4 & 1 \\
\hline
\end{tabular}

ECAM = eventos cardíacos adversos maiores; IAM = infarto agudo do miocárdio; $\mathrm{n}=$ número de pacientes; RVA = revascularização do vaso-alvo.

TABELA 3

Preditores independentes de mortalidade para todos os pacientes

\begin{tabular}{lccc}
\hline & HR ajustado & IC 95\% & $\mathbf{p}$ \\
\hline Idade & 1,05 & $1,04-1,06$ & $<0,01$ \\
Diabetes & 1,27 & $1,10-1,48$ & $<0,01$ \\
DAC triarterial & 1,46 & $1,10-1,94$ & $<0,01$ \\
ICC & 2,20 & $1,62-2,99$ & $<0,01$ \\
Diálise & 5,06 & $2,05-12,49$ & $<0,01$
\end{tabular}

$\overline{\mathrm{DAC}}=$ doença arterial coronária; $\mathrm{HR}=$ hazard ratio; IC 95\% = intervalo de confiança de 95\%; ICC = insuficiência cardíaca congestiva.

O desfecho combinado de eventos cardíacos adversos maiores (soma de óbito, infarto e revascularização do vaso-alvo) foi semelhante nas duas amostras (agudos 11,9\% vs. estáveis 9,9\%; p =0,4). Esse achado, no entanto, merece explanação pormenorizada.

A eficácia dos stents farmacológicos, medida pelo baixo índice de revascularização do vaso tratado, ficou bem evidente nesta série. Seguimos uma população com co-morbidades muitas vezes excluídas de estudos randomizados (por exemplo, insuficiência renal dialítica, acidente vascular cerebral, disfunção ventricular, etc.) e sem qualquer critério de exclusão angiográfica. Ainda assim, independentemente do diagnóstico de admissão, os índices de revascularização do 
vaso-alvo ficaram abaixo de dois dígitos e não alcançaram diferença estatisticamente significante entre os grupos (agudos $7,7 \%$ vs. estáveis $6 \% ; p=0,4$ ). Os resultados obtidos nessa população brasileira são semelhantes aos encontrados em grandes registros internacionais de "mundo real", em que novas revascularizações do vaso tratado ficam perto de $3 \%$ a $11 \%{ }^{15,16}$.

Os índices de óbito semelhantes entre pacientes agudos e crônicos $(6,5 \%$ vs. $4,5 \% \%$, respectivamente; $\mathrm{p}=0,2)$ demonstram que, por um lado, nessa população, o impacto da síndrome instável na utilização dos stents eluidores não pareceu expor os pacientes a maior mortalidade. No entanto, os quadros coronários agudos sem supradesnível de ST, por si, deveriam representar maior risco em relação à angina estável. A semelhança nesse desfecho pode ser explicada pelo fato de a maioria dos pacientes ter sido admitida com angina instável e não com infarto.

A ocorrência de novos eventos isquêmicos agudos, analisada por meio de novos infartos, foi quase o dobro no grupo agudo, mas não alcançou significância estatística $(2,8 \%$ vs. $5 \% ; p=0,1)$. Ao se analisar a trombose de stent pelos critérios do Academic Research Consortium, observa-se ocorrência global de $2,3 \%$, semelhante aos índices descritos na literatura para esse tipo de paciente ${ }^{17}$. No entanto, à avaliação separada dos grupos, pudemos observar que a trombose do stent foi mais freqüente nos pacientes com angina instável/infarto do miocárdio sem supra de ST $(4,4 \%$ vs. $1,4 \% ; p=0,01)$, dado corroborado pelos estudos que já identificaram as síndromes coronárias agudas como preditor independente de trombose dos stents farmacológicos ${ }^{17,18}$. Entre os eventos do grupo agudo, observamos que a maioria das tromboses $(2,9 \%)$ ocorreu entre trinta dias e um ano. Esses dados reforçam os achados dos estudos PCI-CURE (Percutaneous Coronary Intervention - The Clopidogrel in Unstable Angina to Prevent Recurrent Events Trial) e CREDO (Clopidogrel for Reduction of Events During Observation), em que portadores de síndromes coronárias agudas tiveram redução de óbito e eventos isquêmicos com a utilização prolongada de nove a doze meses da terapia antiplaquetária dupla (ácido acetilsalicílico + clopidogrel) $)^{19,20}$. A mediana do tempo de utilização de terapia antiplaquetária dupla nessa população foi de 180 dias. Vale ressaltar, porém, que esta série data do período em que o FDA (Food and Drug Administration) recomendava a utilização da associação do clopidogrel por três (stent Cypher ${ }^{\circledR}$ ) a seis meses (stent Taxus ${ }^{\circledR}$ ), recomendações que em dezembro de 2006 foram mudadas para utilização de pelo menos um ano em pacientes com baixo risco de sangramento. É sabido que a trombose do stent possui, muitas vezes, conseqüências catastróficas, com oclusão aguda do vaso ou morte súbita. Como as tromboses ocorreram com freqüência relativamente baixa e como nem todas as tromboses se transfizeram em óbito, o incremento em novos eventos isquêmicos nos agudos não se refletiu em maior mortalidade.

\section{CONCLUSÕES}

Os stents farmacológicos demonstraram bom perfil de segurança nos pacientes com síndromes coronárias agudas em relação àqueles portadores de quadros clínicos estáveis, apesar da maior ocorrência de trombose tardia do stent.

\section{REFERÊNCIAS BIBLIOGRÁFICAS}

1. Morice MC, Serruys PW, Sousa JE, Fajadet J, Ban Hayashi E, Perin $M$, et al. A randomized comparison of a sirolimuseluting stent with a standard stent for coronary revascularization. N Engl J Med. 2002;346(23):1773-80.

2. Moses JW, Leon MB, Popma JJ, Fitzgerald PJ, Holmes DR, $\mathrm{O}^{\prime}$ Shaughnessy $\mathrm{C}$, et al. Sirolimus-eluting stents versus standard stents in patients with stenosis in a native coronary artery. N Engl J Med. 2003;349(14):1315-23.

3. Schampaert E, Cohen EA, Schlüter M, Reeves F, Traboulsi M, Title LM, et al. The Canadian study of the sirolimuseluting stent in the treatment of patients with long de novo lesions in small native coronary arteries (C-SIRIUS). J Am Coll Cardiol. 2004;43(6):1110-5.

4. Schofer J, Schlüter M, Gershlick AH, Wijns W, Garcia E, Schampaert E, et al. Sirolimus-eluting stents for treatment of patients with long atherosclerotic lesions in small coronary arteries: double-blind, randomised controlled trial (E-SIRIUS). Lancet. 2003;362(9390):1093-9.

5. Grube E, Silber S, Hauptmann KE, Mueller R, Buellesfeld L, Gerckens U, et al. TAXUS I: six- and twelve-month results from a randomized, double-blind trial on a slow-release paclitaxel-eluting stent for de novo coronary lesions. Circulation. 2003;107(1):38-42.

6. Colombo A, Drzewiecki J, Banning A, Grube E, Hauptmann K, Silber S, et al. Randomized study to assess the effectiveness of slow-and moderate-release polymer-based paclitaxeleluting stents for coronary artery lesions. Circulation. 2003; 108(7):788-94.

7. Stone GW, Ellis SG, Cox DA, Hermiller J, O'Shaughnessy C, Mann JT, et al. A polymer-based, paclitaxel-eluting stent in patients with coronary artery disease. N Engl J Med. 2004; 350(3):221-31.

8. Stone GW, Ellis SG, Cox DA, Hermiller J, O'Shaughnessy C, Mann JT, et al. One-year clinical results with the slowrelease, polymer-based, paclitaxel-eluting TAXUS stent: the TAXUS-IV trial. Circulation. 2004;109(16):1942-7.

9. Stone GW, Ellis SG, Cannon L, Mann JT, Greenberg JD, Spriggs D, et al. Comparison of a polymer-based paclitaxeleluting stent with a bare metal stent in patients with complex coronary artery disease: a randomized controlled trial. JAMA. $2005 ; 294(10): 1215-23$.

10. Park DW, Park SW, Park KH, Lee BK, Kim YH, Lee CW, et al. Frequency of and risk factors for stent thrombosis after drug-eluting stent implantation during long-term follow-up. Am J Cardiol. 2006;98(3):352-6.

11. Serruys PW, Daemen J. Are drug-eluting stents associated with a higher rate of late thrombosis than bare metal stents? Late stent thrombosis: a nuisance in both bare metal and drug-eluting stents. Circulation. 2007;115(11):1433-9.

12. Ambrose JA, Winters SL, Stern A, Eng A, Teichholz LE, Gorlin R, et al. Angiographic morphology and the pathogenesis of unstable angina pectoris. J Am Coll Cardiol. $1985 ; 5(3): 609-16$. 
13. Dangas G, Mehran R, Wallenstein S, Courcoutsakis NA, Kakarala V, Hollywood J, et al. Correlation of angiographic morphology and clinical presentation in unstable angina. J Am Coll Cardiol. 1997;29(3):519-25.

14. Chen L, Crook JR, Tousoulis D, Chester MR, Kaski JC. Complex stenosis morphology predicts late reocclusion during follow-up after myocardial infarction in patients with patent infarct-related coronary arteries. Am Heart J. 1998; 136(5):877-83.

15. Ong AT, van Domburg RT, Aoki J, Sonnenschein K, Lemos PA, Serruys PW. Sirolimus-eluting stents remain superior to bare-metal stents at two years: medium-term results from the Rapamycin-Eluting Stent Evaluated at Rotterdam Cardiology Hospital (RESEARCH) registry. J Am Coll Cardiol. 2006; 47(7):1356-60.

16. Marzocchi A, Saia F, Piovaccari G, Manari A, Aurier E, Benassi A, et al. Long-term safety and efficacy of drug-eluting stents: two-year results of the REAL (REgistro AngiopLastiche dell'Emilia Romagna) multicenter registry. Circulation. 2007; $115(25): 3181-8$
17. Daemen J, Wenaweser P, Tsuchida K, Abrecht L, Vaina S, Morger C, et al. Early and late coronary stent thrombosis of sirolimus-eluting and paclitaxel-eluting stents in routine clinical practice: data from a large two-institutional cohort study. Lancet. 2007;369(9562):667-78.

18. Urban P, Gershlick AH, Guagliumi G, Guyon P, Lotan C, Schofer J, et al. e-Cypher Investigators. Safety of coronary sirolimus-eluting stents in daily clinical practice: one-year follow-up of the e-Cypher registry. Circulation. 2006; 113(11):1434-41.

19. Yusuf S, Zhao F, Mehta SR, Chrolavicius S, Tognoni G, Fox $\mathrm{KK}$, et al, Clopidogrel in Unstable Angina to Prevent Recurrent Events Trial Investigators. Effects of clopidogrel in addition to aspirin in patients with acute coronary syndromes without STsegment elevation. N Engl J Med. 2001;345(7):494-502.

20. Mehta SR, Yusuf S, Peters RJ, Bertrand ME, Lewis BS, Natarajan $M K$, et al. Effects of pretreatment with clopidogrel and aspirin followed by long-term therapy in patients undergoing percutaneous coronary intervention: the PCI-CURE study. Lancet. $2001 ; 358(9281): 527-33$. 